\title{
EXTRAÇÃO E EXPORTAÇÃO DE NUTRIENTES EM FRUTOS DE GRAVIOLEIRA
}

\author{
Gedeon Almeida Gomes Júnior ${ }^{1,}$ Rafaela Alves Pereira² ${ }^{2}$ Célio Kersul do Sacramento ${ }^{3}$, José Olímpio de Souza Júnior ${ }^{3}$
}

\author{
${ }^{1}$ MSc em Produção Vegetal, Universidade Estadual de Santa Cruz, Ilhéus-Ba \\ ${ }^{2} \mathrm{DSc}$ em Produção Vegetal, Universidade Estadual de Santa Cruz, Ilhéus-Ba \\ ${ }^{3}$ Professor adjunto, Universidade Estadual de Santa Cruz, Ilhéus-Ba
}

Autor para correspondência: Gedeon Almeida Gomes Júnior, geda_almeida@hotmail.com

\begin{abstract}
RESUMO: Em diversas espécies frutíferas, a quantidade de nutrientes exportada pelos frutos vem sendo utilizada como guia para nortear um bom programa de adubação. São raros os trabalhos científicos realizados sobre a absorção dos nutrientes minerais em várias espécies frutíferas, como a graviola. 0 objetivo desse trabalho foi avaliar a composição mineral dos frutos de gravioleira e quantificar a extração de macro e micronutrientes da cultura. Para isso, foram coletados ao acaso 40 frutos de gravioleira no município de llhéus (Bahia) e separados em casca, polpa, placenta e semente. Foi realizada estatística descritiva para obtenção da média e coeficiente de variação. A ordem crescente de magnitude dos elementos extraídos em frutos de gravioleira foi $\mathrm{K}>\mathrm{N}>\mathrm{Ca}$ $>\mathrm{P}>\mathrm{Mg}$ para os macronutrientes, e $\mathrm{Fe}>\mathrm{Zn}>\mathrm{Cu}>\mathrm{Mn}$, para os micronutrientes. A exportação de nutrientes na polpa dos frutos de graviola foi superior às exportações na casca, semente e placenta. A ordem de magnitude para exportação de nutrientes em frutos inteiros foi $\mathrm{K}>\mathrm{N}>\mathrm{P}>\mathrm{Ca}>\mathrm{Mg}>\mathrm{Fe}>\mathrm{Zn}>\mathrm{Cu}>\mathrm{Mn}$. A exportação de nutrientes por hectare para os macronutrientes $\mathrm{K}, \mathrm{N}, \mathrm{Ca}, \mathrm{P}$ e Mg foi de 16,26; 12,87; 2,05; 1,80; e 1,44 kg, respectivamente. Dentre os micronutrientes, as exportações de Fe, Zn, Cu e Mn para um hectare foram 147,82; 28,68; 23,00 e 16,55 g, respectivamente.
\end{abstract}

PALAVRAS-CHAVE: Annona muricata L., partição, nutrição de plantas

\section{NUTRIENT EXTRACTION AND EXPORT BY SOURSOP FRUITS}

\begin{abstract}
In several fruit species, the amount of nutrients exported by the fruits has been used as a guide to guide a good fertilization program. Scientific studies on the absorption of mineral nutrients in various fruit species, such as soursop, are rare. The objective of this work was to evaluate the mineral composition of soursop fruits and to quantify the extraction of macro and micronutrients in the fruits. For this, 40 fruits of soursop were harvested in the municipality of Ilhéus - Bahia and separated in bark, pulp, placenta and seed. Descriptive statistics were obtained to obtain the statistical parameters, mean and coefficient of variation. The increasing order of magnitude of the elements extracted in sage fruits was $\mathrm{K}>\mathrm{N}>\mathrm{Ca}>\mathrm{P}>\mathrm{Mg}$ for the macronutrients, and $\mathrm{Fe}>\mathrm{Zn}>\mathrm{Cu}>\mathrm{Mn}$, for the micronutrients. The export of nutrients in the pulp of soursop fruits was superior to exports in the bark, seed and placenta. The order of magnitude for nutrient exportation in whole fruits was $\mathrm{K}>\mathrm{N}>\mathrm{P}>\mathrm{Ca}>\mathrm{Mg}>\mathrm{Fe}>\mathrm{Zn}>\mathrm{Cu}>\mathrm{Mn}$. The nutrient exportation per hectare for the macronutrients K, N, Ca, P and Mg was $16.26 \mathrm{~kg} ; 12.87 \mathrm{~kg} ; 2.05 \mathrm{~kg}$; $1.80 \mathrm{~kg}$; and $1.44 \mathrm{~kg}$ respectively. Among the micronutrients, exports to $\mathrm{Fe}, \mathrm{Zn}, \mathrm{Cu}$ and $\mathrm{Mn}$ for one hectare 147.82 $\mathrm{g} ; 28.68 \mathrm{~g} ; 23.00 \mathrm{~g}$ and $16.55 \mathrm{~g}$ respectively.
\end{abstract}

KEY WORDS: Annona muricata L., partition, plant nutrition

\section{INTRODUÇÃO}

O nordeste brasileiro apresenta condições favoráveis ao cultivo de várias espécies frutíferas. A gravioleira (Annona muricata L.) é considerada a fruta mais tropical na família das anonáceas, sendo cultivada em diversos países da América, África e Ásia. No Brasil, é cultivada principalmente nos
Estados da Paraíba, Alagoas, Ceará, Pernambuco, Minas Gerais, Pará, Distrito Federal e Bahia (Sacramento et al., 2009). Na região sul do estado da Bahia, devido à implantação de agroindústrias, o cultivo da graviola encontra-se em expansão com crescente demanda pela polpa que é a quinta mais vendida, com $12 \%$ do total, ficando em volume atrás 
apenas das polpas de acerola, goiaba, maracujá e caju (Lemos, 2014).

O levantamento do estado nutricional das culturas tem sido bastante pesquisado e utilizado, baseado especialmente na interpretação da análise de tecido vegetal e do solo, buscando ações para melhorar a produtividade vegetal pela adubação (Rozane e Natale, 2014). No entanto, em plantas adultas, onde frutos são retirados do pomar para posterior comercialização, a reposição de nutrientes ao solo não deve ser realizada apenas mediante adubação com base em análises do solo e foliar, considerando que em espécies frutíferas, a extração e consequentemente, a exportação de nutrientes ocorre de maneira frequente (São José et al., 2014). Dessa forma, o conhecimento das necessidades nutricionais de uma cultura é essencial para recomendação da adubação, através da estimativa do acúmulo de nutrientes nas folhas, nos frutos e pela extração realizada na colheita (Laviola e Dias, 2008).

Em diversas espécies frutíferas, a quantidade de nutrientes exportada pelos frutos vem sendo utilizada como um guia para nortear um bom programa de adubação. As anonáceas, apesar da aparente rusticidade, extraem do solo grande quantidade de elementos minerais e essa extração pode variar em função da espécie botânica, fase de crescimento e desenvolvimento, além das condições ambientais ao longo do ano (São José et al., 2014).

Apesar da importância da nutrição mineral para diversas espécies frutíferas, raros são os trabalhos científicos realizados sobre a absorção dos nutrientes minerais. Levando em conta que a graviola é uma fruta com crescente demanda de nutrientes, o objetivo do presente trabalho foi avaliar a composição mineral dos frutos e quantificar a extração de macro e micronutrientes visando subsidiar estudos para a reposição de nutrientes no programa de adubação da cultura.

\section{MATERIAL E MÉTODOS}

0 experimento foi realizado no município de Ilhéus (Bahia), em agosto de 2017. Foram selecionadas 10 plantas matrizes em pomar localizado no município de Ilhéus. Foram retirados 4 frutos por planta e separados em casca, polpa, placenta e semente. Foram pesados para obtenção da matéria fresca e levados a estufa de ventilação à $105^{\circ} \mathrm{C}$ por 72 horas. Em seguida, as amostras foram pesadas em balança analítica com precisão de 0,01 g para obtenção da matéria seca das amostras.

As concentrações dos nutrientes foram determinadas por digestão nítrica-perclórica e digestão sulfúrica para $\mathrm{N}$. Os teores de $\mathrm{P}$ foram determinados pelo método do molibdato, em espectrofotômetro de absorção molecular; $\mathrm{K}$ por fotometria de chama; $\mathrm{Ca}, \mathrm{Mg}, \mathrm{Cu}, \mathrm{Fe}, \mathrm{Mn}$ e $\mathrm{Zn}$ por espectrometria de absorção atômica, e $\mathrm{N}$ pelo método micro Kjeldahl (Silva, 2009).

0 acumulo de macro e micronutrientes na casca, polpa, semente e placenta dos frutos foi calculado pela relação entre a matéria seca e a concentração do nutriente. De posse dos valores do acumulo para cada nutriente estimou-se a exportação de nutrientes por hectare, assumindo uma produtividade $7000 \mathrm{t}$ de frutos frescos. Foi realizada estatística descritiva para obtenção dos parâmetros estatísticos, média e coeficiente de variação.

\section{RESULTADOS E DISCUSSÃO}

Os teores de umidade dos frutos foram obtidos pela relação entre massa fresca e massa seca das amostras (Tabela 1). A concentração de macronutrientes na casca, polpa, semente e placenta obedeceram à seguinte ordem: $\mathrm{K}>\mathrm{N}>\mathrm{Ca}>\mathrm{P}>\mathrm{Mg} ; \mathrm{K}>\mathrm{N}>\mathrm{P}>\mathrm{Ca}$ $>\mathrm{Mg}$; $>\mathrm{K}>\mathrm{P}>\mathrm{Mg}>\mathrm{Ca}$ e $\mathrm{K}>\mathrm{N}>\mathrm{P}>\mathrm{Mg}>\mathrm{Ca}$, respectivamente. Já os micronutrientes nesses mesmos compartimentos obedeceram a seguinte ordem: $\mathrm{Fe}>$ $\mathrm{Cu}>\mathrm{Mn}>\mathrm{Zn}$; $\mathrm{Fe}>\mathrm{Zn}>\mathrm{Cu}>\mathrm{Mn}$; $\mathrm{Fe}>\mathrm{Zn}>\mathrm{Mn}>\mathrm{Cu} e$ $\mathrm{Fe}>\mathrm{Zn}>\mathrm{Cu}>\mathrm{Mn}$, respectivamente (Tabela 2).

$\mathrm{O} \mathrm{K}$ foi o macronutriente que apresentou os maiores teores na casca $\left(14,1 \mathrm{~g} \mathrm{~kg}^{-1}\right)$, polpa $(13,28 \mathrm{~g} \mathrm{~kg}$ 1) e placenta $\left(22,14 \mathrm{~g} \mathrm{~kg}^{-1}\right)$ de frutos de gravioleira constituindo juntos $90 \%$ da matéria seca dos frutos (Tabela 1). Cruz et al. (2013), avaliando os teores de nutrientes em frutos de atemóia (Annona squamosa L.), também relataram que o $\mathrm{K}$ foi o nutriente com maior teor em frutos. Sodré et al. (2012) relatam uma concentração de $145,9 \mathrm{~g} \mathrm{~kg}^{-1}$ no extrato de casca de cacau. 0 potássio desempenha importante papel na formação dos frutos, atuando no transporte de fotoassimilados no floema. (Marschner, 2012), o que no caso explicaria o seu maior teor nos frutos. Dessa forma, nas anonáceas em geral, esse elemento é exigido durante a fase de formação de frutos de maneira significativa para a síntese de carboidratos e outros compostos, devendo este estar disponível em quantidades adequadas (São José et al., 2014). 
Tabela 1. Matéria fresca e matéria seca dos frutos e suas respectivas partes.

\begin{tabular}{lcccc}
\hline Partes & Matéria Fresca & Matéria seca & Umidade & Proporção do fruto \\
& -1 & & $-\cdots$ & 14 \\
\hline Casca & 488,8 & 192,06 & 60,7 & 2 \\
Placenta & 75,2 & 14,25 & 81,05 & 79 \\
Polpa & 2811,05 & 425,03 & 84,88 & 5 \\
Semente & 179,12 & 112,07 & 37,43 & 100 \\
\hline Fruto & 3554,2 & 743,41 & 79,08 & 0 \\
\hline
\end{tabular}

$\mathrm{O} \mathrm{N}$ foi o segundo elemento mais requerido pelos frutos, apresentando os maiores teores na semente $(18,19 \mathrm{~g} \mathrm{~kg}$ 1) e correspondendo em média a $10 \%$ da matéria seca dos frutos (Tabela 2). O N está diretamente envolvido no florescimento e frutificação das anonáceas, dessa forma o fornecimento desequilibrado deste nutriente pode limitar o potencial produtivo da cultura. (São José et al., 2014). No entanto, não se encontra na literatura informações sobre exportação desse nutriente por frutos de A. muricata.

Tabela 2. Teores médios de macronutrientes e micronutrientes em frutos de gravioleira.

\begin{tabular}{|c|c|c|c|c|c|c|c|c|c|}
\hline \multirow{2}{*}{ Partes } & \multirow[t]{2}{*}{$\mathbf{N}$} & $\mathbf{P}$ & $\mathrm{K}$ & $\mathrm{Ca}$ & \multirow[t]{2}{*}{$\mathrm{Mg}$} & \multirow[t]{2}{*}{$\mathrm{Fe}$} & $\mathrm{Zn}$ & $\mathrm{Cu}$ & \multirow[t]{2}{*}{$\mathrm{Mn}$} \\
\hline & & \multicolumn{3}{|c|}{---g kg-1 } & & & \multicolumn{2}{|c|}{ 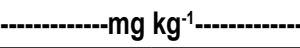 } & \\
\hline Casca & 9,4 & 1,34 & 14,17 & 1,7 & 1,01 & 59,82 & 7,06 & 16,16 & 13,93 \\
\hline CV $(\%)$ & 8,22 & 12,59 & 17,39 & 17,77 & 11,06 & 25,02 & 15,14 & 16,82 & 10,86 \\
\hline Placenta & 17,53 & 4,04 & 22,14 & 2,38 & 4,22 & 44,33 & 21,74 & 19,82 & 17,07 \\
\hline CV $(\%)$ & 11,3 & 17,68 & 18,87 & 23,89 & 14,11 & 11,98 & 36,57 & 13,9 & 6,76 \\
\hline Polpa & 9,14 & 1,59 & 13,29 & 1,29 & 1,06 & 157,73 & 28,91 & 19,16 & 11,22 \\
\hline CV (\%) & 9,35 & 19,67 & 16,18 & 24,29 & 12,27 & 6,08 & 15,19 & 9,59 & 14,84 \\
\hline Semente & 18,19 & 2,25 & 5,19 & 1,36 & 1,44 & 47,76 & 30,63 & 18,85 & 22,56 \\
\hline CV (\%) & 14,25 & 20,33 & 22,11 & 22,99 & 12,44 & 9,52 & 13,51 & 15,17 & 9,79 \\
\hline
\end{tabular}

$\mathrm{CV}=$ coeficiente de variação.

O Ca vem sendo relatado como importante nutriente na fisiologia de diversas espécies de anonáceas, favorecendo desde o crescimento vegetativo a formação do fruto, sendo indispensável da formação até a maturidade fisiológica dos frutos. Em relação a $\mathrm{K}$ e N, os teores de Ca foram bem inferiores no presente estudo (Tabela 3). Isso pode ser explicado por este nutriente apresentar reduzida redistribuição da folha para o fruto, de forma que o seu transporte ocorre preferencialmente para locais de maior transpiração e de maneira unidirecional das raízes até a parte aérea. Além disso, deve-se considerar que $\mathrm{K}$ e $\mathrm{N}$ são nutrientes mais móveis no floema, podendo ser facilmente remobilizados de um órgão-fonte para órgãos-dreno (Aular e Natale, 2013; Marschner, 2011; Mengel e Kirkby, 2001).

Tabela 3. Exportação de macronutrientes e micronutrientes em frutos de gravioleira.

\begin{tabular}{|c|c|c|c|c|c|c|c|c|c|}
\hline \multirow{2}{*}{ Partes } & $\mathbf{N}$ & $\mathbf{P}$ & $\mathrm{K}$ & $\mathrm{Ca}$ & $\mathrm{Mg}$ & $\mathrm{Fe}$ & $\mathrm{Zn}$ & $\mathrm{Cu}$ & $\mathrm{Mn}$ \\
\hline & \multicolumn{5}{|c|}{ - } & \multicolumn{4}{|c|}{ 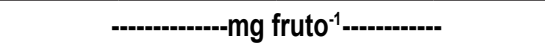 } \\
\hline Casca & 1,81 & 0,26 & 2,72 & 0,33 & 0,19 & 11,49 & 1,36 & 3,1 & 2,68 \\
\hline Placenta & 0,25 & 0,06 & 0,32 & 0,03 & 0,06 & 0,63 & 0,31 & 0,28 & 0,24 \\
\hline Polpa & 3,89 & 0,68 & 5,65 & 0,55 & 0,45 & 67,04 & 12,29 & 8,15 & 4,77 \\
\hline Semente & 2,04 & 0,25 & 0,58 & 0,15 & 0,16 & 5,35 & 3,43 & 2,11 & 2,53 \\
\hline Fruto & 7,98 & 1,24 & 9,27 & 1,06 & 0,86 & 84,51 & 17,39 & 13,64 & 10,22 \\
\hline
\end{tabular}

O P ficou entre os macronutrientes acumulados em menor quantidade nos frutos de graviola, $(1,24$ $\mathrm{g}$ fruto $^{-1}$ ) (Tabela 3). Comportamento semelhante foi observado por Aguiar Neto et al. (2016) em frutos de melancia, onde a exportação de $\mathrm{P}$ também foi inferior a K e N. Destaca-se ainda, que a taxa de redistribuição do nutriente no fruto está relacionada à mobilidade de cada nutriente (Maillard et al., 2015). 
A exportação de nutrientes por fruto obedeceu à seguinte ordem $\mathrm{K}>\mathrm{N}>\mathrm{P}>\mathrm{Ca}>\mathrm{Mg}$ para os macronutrientes e $\mathrm{Fe}>\mathrm{Zn}>\mathrm{Cu}>\mathrm{Mn}$ para os micronutrientes (Tabela 3). A mesma sequência foi observada para exportação em Kg ha-1 (Tabela 4). A maior quantidade de nutriente das frutas se concentra nas cascas e sementes (Melo et al., 2008; Abrahão et al., 2010). O mesmo ocorreu no presente estudo, onde maiores teores de macro e micronutriente foram observados na casca (Tabela 4), a exemplo do K, que apresentou no compartimento casca, em média 30\% do total exportado por fruto. Almeida et al. (2009) relataram que o $\mathrm{K}$ é o nutriente mais abundante em frutas tropicais cultivadas no nordeste brasileiro.

Tabela 4. Exportação estimada de macronutrientes e micronutrientes de frutos de graviola para uma produtividade de 7000 tha de frutos frescos.

\begin{tabular}{|c|c|c|c|c|c|c|c|c|c|}
\hline \multirow{2}{*}{ Partes } & \multirow[t]{2}{*}{$\mathbf{N}$} & $\mathbf{P}$ & $\mathrm{K}$ & $\mathrm{Ca}$ & \multirow[t]{2}{*}{$\mathrm{Mg}$} & \multirow[t]{2}{*}{$\mathrm{Fe}$} & $\mathrm{Zn}$ & $\mathrm{Cu}$ & \multirow[t]{2}{*}{$\mathrm{Mn}$} \\
\hline & & \multicolumn{3}{|c|}{ - } & & & \multicolumn{2}{|c|}{ 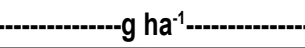 } & \\
\hline Casca & 3,3 & 0,47 & 4,97 & 0,59 & 0,35 & 20,97 & 2,47 & 5,66 & 4,88 \\
\hline Placenta & 0,45 & 0,1 & 0,56 & 0,06 & 0,11 & 1,13 & 0,55 & 0,50 & 0,43 \\
\hline Polpa & 6,96 & 1,21 & 10,11 & 0,98 & 0,80 & 120,03 & 22,0 & 14,58 & 8,54 \\
\hline Semente & 2,17 & 0,27 & 0,62 & 0,16 & 0,17 & 5,69 & 3,65 & 2,25 & 2,69 \\
\hline Total & 12,87 & 2,05 & 16,26 & 1,80 & 1,44 & 147,82 & 28,68 & 23,00 & 16,55 \\
\hline
\end{tabular}

Este resultado concorda e é similar ao de vários outros trabalhos, os quais versam sobre exigência nutricional em outras culturas. $\mathrm{K}$ e Fe foram, respectivamente, 0 macro e micronutriente mais exportados em frutos de mamoeiro em plena maturidade fisiológica tratados com biofertilizantes (Mesquita et al., 2010). $0 \mathrm{~K}$ também foi 0 macronutriente mais exportado em frutos de melancia (Aguiar Neto et al., 2016). A maior magnitude de exportação de $K$ para essas culturas está relacionada ao papel que este elemento exerce no transporte de solutos solúveis como também na manutenção de um elevado teor de água no fruto (Cardoso et al., 2011).

Em relação ao micronutriente Fe, Araújo et al. (2016) trabalhando com frutos de meloeiro relataram comportamento similar ao verificado no presente estudo. Esses mesmo autores observaram que o fruto é o dreno preferencial deste micronutriente. Isso pode perfeitamente ser relacionado ao elevado acúmulo de Fe nos frutos de graviola.

Em zonas produtoras de graviola, observa-se que plantas bem nutridas apresentam produção continuada ao longo do ano e que solos com adequada disponibilidade de nutrientes favorece o crescimento vegetativo e reprodutivo (São José et al., 2014). No que se refere à nutrição mineral da gravioleira, sabe-se que o $\mathrm{K}$ e $\mathrm{N}$ são os elementos mais requeridos, com elevadas demandas desde a fase vegetativa até a frutificação. Esses mesmos nutrientes foram os mais exportados pelos frutos.
Devido as cascas e sementes serem descartadas logo após o beneficiamento, o surgimento de resíduo agroindústrias tem aumentado nos últimos anos (Souza et al., 2011) e esses mesmos resíduos demonstram potencial para reaproveitamento em práticas como a compostagem, por converter resíduos orgânicos em compostos capazes de reciclar nutrientes no solo (Domínguez e Gómez-Brandón, 2010), como também, apresentam potencial para composição da dieta alimentar de animais. Silva et al. (2014), avaliando a composição de resíduo agroindustrial composto da casca e sementes de frutos de gravioleira, relataram potencial destes na composição da dieta alimentar de ruminantes como alimentos volumosos.

Desta forma pode-se concluir que: a ordem crescente de magnitude dos elementos extraídos em frutos de gravioleiraé $\mathrm{K}>\mathrm{N}>\mathrm{Ca}>\mathrm{P}>$ Mg para os macronutrientes, e $\mathrm{Fe}>\mathrm{Zn}>\mathrm{Cu}>\mathrm{Mn}$, para os micronutrientes.

A exportação de nutrientes na polpa dos frutos de graviola é superior às exportações na casca, semente e placenta dos frutos.

A ordem de magnitude para exportação de nutrientes em frutos inteiros é $\mathrm{K}>\mathrm{N}>\mathrm{P}>\mathrm{Ca}>\mathrm{Mg}>$ $\mathrm{Fe}>\mathrm{Zn}>\mathrm{Cu}>\mathrm{Mn}$.

\section{REFERÊNCIAS BIBLIOGRÁFICAS}

Abrahão, S.A.; Pereira, R.G.F.A.; Duarte, S.M.S.; Lima, A.R.; Alvarenga, D.J.; Ferreira, E.B. Compostos bioativos e atividade antioxidante do café (Coffea arabica L.). Ciência e Agrotecnologia. 2010, 34, 2, 414-420. 
Aguiar Neto, P.; Souza, R.A.G.; Maracajá, P.B.; Medeiros, A.C.; Pimenta, T.A.; Lima, T.S. Crescimento e absorção de macronutrientes na cultura da melancia no estado de Pernambuco. Revista Verde de Agroecologia e Desenvolvimento Sustentável. 2016, 11, 4, 17-25.

Almeida, M.M.B.; Sousa, P.H.M.; Fonseca, M.L.; Magalhães, C.E.C.; Lopes, M.F.G.; Lemos, T.L.G. Evaluation of macro and micro-mineral content in tropical fruits cultivated in the northeast of Brazil. Ciência e Tecnologia de Alimentos. 2009, 29, 581-586.

Aular, J.; Natale, W. Nutrição mineral e qualidade do fruto de algumas frutíferas tropicais: goiabeira, mangueira, bananeira e mamoeiro. Revista Brasileira de Fruticultura. 2013, 35, 4, 1214-1231.

Araújo, J.L.; Oliveira, F.S. de; Oliveira, F.S. de. Partição de nutrientes na parte aérea do meloeiro 'Goldex' fertirrigado. Revista Agro@mbiente On-line. 2016, 10, 4, 299-308.

Cardoso, E.A.; Costa, J.T.A.; Soares, I.; Silva, R.M. da; Aguiar, A.V.M. de. Exportação de nutrientes por frutos de goiabeira "paluma" em função da adubação mineral. Agropecuária científica no semiárido. 2011, 6, 3, 38-41.

Cruz, L.; Lima, R.; Abreu, C.; Corrêa, A.; Pinto, L. Physical and chemical characterization of fractions of fruit atemoya Gefner. Ciência Rural. 2013, 43, 12 , 2280-2284.

Domínguez, J.; Gómez-Brandón, M. Ciclos de vida de laslombrices de tierra aptas para el vermicompostaje. Acta Zoológica Mexicana. 2010, 26, esp.2, 309 - 320.

Laviola, B.G.; Dias, L.A.S. Teor e acúmulo de nutrientes em folhas e frutos de pinhão-manso. Revista Brasileira de Ciência do Solo. 2008, 32, 5, 1969-1975.

Lemos, E.E.P. de. A produção de anonáceas no Brasil. Revista Brasileira de Fruticultura. 2014, 36, esp.1, 77-85.

Maillard, A.; Billard, V.; Laîné, P.; Garnica, M.; Prudent, M.; Garcia-Mina, J-M.; Yvin, J-C.; Ourry, A. Leaf mineral nutrient remobilization during leaf senescence and modulation by nutrient deficiency. Frontiers in Plant Science. 2015, 6, 1-15.

Marschner, P. Marschner's mineral nutrition of higher plant. 3. ed. Londres: Academic Press. Elsevier. San Diego, USA. 2012.

Melo, E.A.; Maciel, M.I.S.; Lima, V.A.G.L.; Nascimento, R.J. Capacidade Antioxidante de Frutas. Revista Brasileira de Ciências Farmacêuticas. 2008, 2, 193-201.

Mengel, K.; Kirkby, E. Principles of plant nutrition. 5. ed. Dordrecht/Boston/London: Kluwer Academic Publishers, 2001. 849p.

Mesquita, E.F. de; Cavalcante, L.F.; Gondim, S.C.; Campos, V.B.; Cavalcante, Í.H.L.; Gondim, P.C. Teores foliares e exportação de nutrientes do mamoeiro Baixinho de Santa Amália tratado com biofertilizantes. Pesquisa Agropecuária Tropical. 2010, 40, 1, 66-76.

Rozane, D.E.; Natale, W. Calagem, adubação e nutrição mineral de anonáceas. Revista Brasileira de Fruticultura. 2014, 36, 166-175.

Sacramento, C.K. do; Moura, J.I.L.; Coelho Junior, E. Graviola. In: Santos-Serejo, J.A. et al. (eds.). Fruticultura tropical: espécies regionais e exóticas. Brasília, DF: Embrapa Informação Tecnológica. 2009. p. 95-132.

São José, A.R.; Prado, N.B. do; Bomfim, M.P. Marcha de absorção de nutrientes em anonáceas. Revista Brasileira de Fruticultura. 2014, 36, 176-183.

Silva, A.M.; Oliveira, R.L.; Ribeiro, O.L.; Bagaldo, A R.; Bezerra, L.R.; Carvalho, S.T.; Abreu, C.L.; Leão, A.G. Valor nutricional de resíduo da agroindústria para alimentação de ruminantes. Comunicata Scientiae. 2014, 5, 4, 370-379.

Silva, F.C. da. Manual de análises químicas de solos, plantas e fertilizantes. Brasília: EMBRAPA. 2009, 627 p.

Sodré, G.A.; Venturini, M.T.; Ribeiro, D.O.; Marrocos, P.C.L. Extrato da casca do fruto do cacaueiro como fertilizante potássico no crescimento de mudas de cacaueiro. Revista Brasileira de Fruticultura. 2012, 34, 3, 881-887.

Souza, A.D.V.; Fávaro, S.P.; Ítavo, L.C.V.; Roscoe, R. Caracterização química de sementes e tortas de pinhãomanso, naboforrageiro e crambe. Pesquisa Agropecuária Brasileira. 2009, 44, 10, 1328-1335. 